\title{
Functional Property Evaluation of Crystalline Materials using Density Functional Theory: A Review
}

\author{
Naveen Weerasekera, Siyua Cao, and Laksman Perera
}

\begin{abstract}
In this paper, utilization of density functional theory (DFT) to obtain mechanical, electrical and thermal properties of crystalline materials are reviewed. DFT has resulted as an efficient tool for predicting ground states of many body systems thus aiding in resolving dispersion spectrums of complex atomic arrangements where solution by traditional Schrodinger (SH) equation is infeasible. Great success has been reported by previous researchers on utilizing DFT for functional property predictions of crystalline solids.
\end{abstract}

Keywords - Density functional theory, crystalline solid, elastic property, thermal property, electrical property.

\section{INTRODUCTION}

Property prediction of materials is a necessary requirement in materials science. Such properties mainly include mechanical, electronic, and thermal aspects. As the major mechanical property, stress strain behavior is a typical importance. Important electronic and thermal properties at interest are electrical and thermal conductivity, specific heat capacity [1]-[3].

Metals are materials with periodic structures with free electrons. While focusing on electronic and thermal property prediction of metals, the main contribution for both aspects result from electronic contributions, where, for non-crystalline insulator materials, thermal properties arise mainly due to phononic contributions. Therefore, understanding electronic behavior in a crystalline material with free electron dominance is an inherent interest [4] along with phononic dominance in low free electron material. Study of electronic behavior of any material starts from the band structure predictions [5]. Band structure for a material is the fundamental aspect in predicting the electronic energy levels which leads to density of states (DoS) for electronic energy levels. DoS can be further utilized to predict the free electron dominant electrical and thermal property predictions [6].

Density functional theory (DFT) has been an important molecular modeling tool for past few decades [7]. Capability of DFT spans for any material with crystalline nature to predict its inherent properties. It is convenient to solve Schródinger ( $\mathrm{SH}$ ) equation for a single electron system subjected to simple potentials (2-dimensional potential wells, hydrogen atom etc.). However, for more complicated systems, specifically many body systems which involve multiple electrons, solving SH equation is nearly impossible. Therefore, the goal of DFT is to approximate a solution to the many body SH equation, finally providing the ground state energy of the system[8]. Hohenburg and Kohn (HK) theorem can be considered as the main foundation for DFT [7]. HK theorem states that the ground state energy of many electron system is a functional of local electron density. And also, contributions to the total energy of the system which are, ion-electron potential energy, ion-ion potential energy, electron-electron potential energy, kinetic energy and exchangecorrelation energy can be given based on electron density [7], [8].

Focusing on functional property prediction of crystalline materials, DFT as a simulation tool has become highly contributing since its ability to predict the ground state energy of any crystalline system. Approximation processes in DFT allows to reduce complexity of many body problems and aid in efficient property predictions. Fereidoon et al. [9] utilized DFT to obtain mechanical properties of single-walled carbon nanotubes (SW-CNT). They have approached by obtaining ground state energy of the quantum mechanical system by DFT and subsequently applying macroscopic relations to interatomic displacements based on variation of ground state energy under small perturbations. Therefore, mechanical properties of the structure (elastic constant) was obtained based on interatomic displacements. Ajori et al. [10] also utilized DFT to obtain mechanical properties of metal-free two dimensional polymer graphitic carbon nitride. Their approach has also follows the similar pathway as Fereidoon et al. [9]. Chen and Podloucky

Submitted on December 12, 2021

Published on January 13, 2022.

Naveen Weerasekera, Department of Mechanical Engineering, University of Louisville, Louisville, KY, USA

(corresponding e-mail: naveen12.weerasekera@louisville.edu)

Siyua Cao, Department of Mathematics and Statistics, Portland State University, Portland, OR, USA.

Laksman Perera, Department of Physics, Faculty of Science, University of Colombo, Colombo, Sri Lanka. 
[11] utilized DFT to calculate electronic thermal conductivity of crystalline solids. Their work pathed on utilizing Wiedemann-Franz (WF) law which relates electrical conductivity and Lorentz tensor with electronic thermal conductivity. Therefore, obtaining electrical conductivity from DFT is indirectly performed. Silvestrelli [12] used DFT to predict electrical conductivity of liquid sodium. After dispersion relations are obtained from DFT, they have associated Drude formula [13] to arrive at electrical conductivity, since, liquid sodium can be well fitted to the free electron gas approximation. Ranasinghe $e t$ al. [14] used DFT to predict thermal conductivity and specific heat capacity due to phononic contributions. Similarly, dispersion spectrum is resolved by DFT and phonon density of states are utilized for further calculations.

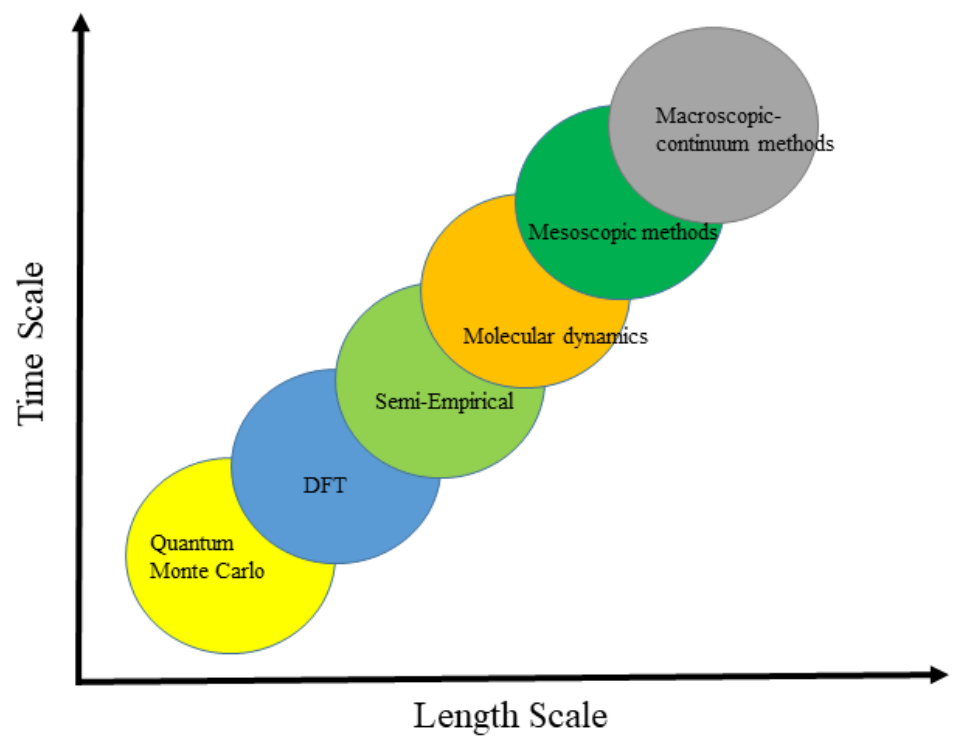

Fig. 1. Simulation time scale of DFT.

\section{A. General Theory of DFT}

Quantum mechanical wave function contains all the information about the given quantum system in principle. To determine the allowed energy of the system, Schrodinger (SH) equation can be solved to obtain the wave function, thus allowing to find the energy states of the system. However, it is infeasible to solve the $\mathrm{SH}$ equation directly for quantum N-body system (Regular crystal) with large number of participants, therefore, solution for SH equations based on approximations are proposed [15]. DFT is such an approximation method to obtain a solution to SH equation for a many body system.

The main approach of DFT is reducing the number of freedoms of the many body system. It is performed by utilizing Bohn-Oppenheimer approximation (BOA). In brief, BOA assumes that the wave functions of atomic nuclei and electrons in a molecule can be treated separately since nuclei is much heavier than electrons. Hohenburg and Kohn (HK) demonstrated that there exist a functional, when minimized over electron densities while integrating to total number of electrons which is distributed over a periodic structure, yields to the exact ground state energy of the system. The exact ground state energy is calculated when number of electrons are subject to an external potential. DFT technique utilize this concept and apply electron density as the functional which is a function of space and time. The ultimate objective of the approach is to develop an effective single electron type $\mathrm{SH}$ equation for the entire system.

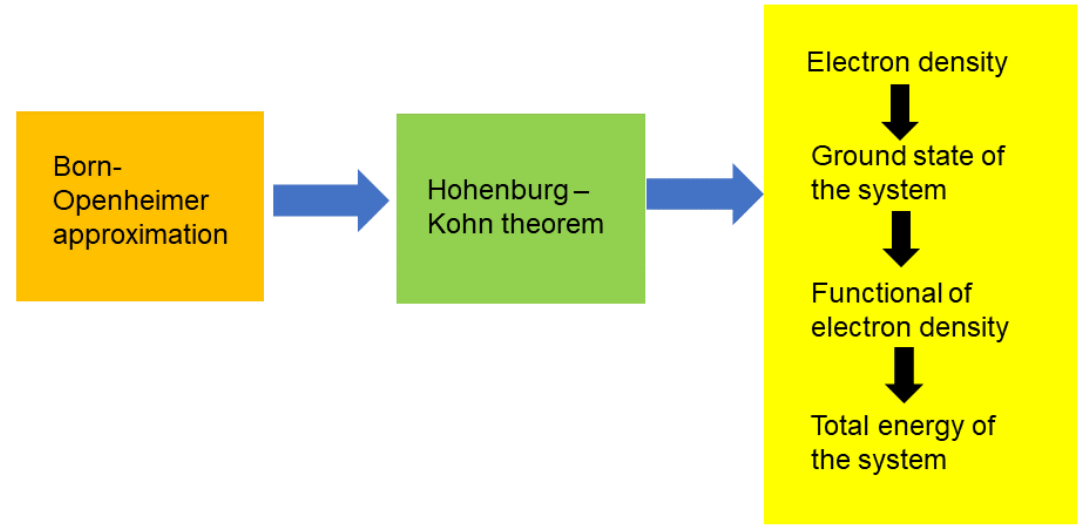

Fig. 2. General roadmap of density functional theory. 
Total energy of the system can now be given considering as a reflection of BOA approach, based on ionion potential energy, ion-electron potential energy, electron-electron potential energy, kinetic energy, and exchange correlation energy [5]. The objective is to develop DFT techniques to evaluate contribution of all such energies as a functional of local electron density [15].

\section{B. Development of Electron Density as a Functional}

Quantum mechanical wave function can be obtained using classical Hamiltonian $(\mathrm{H})$ for $\mathrm{N}$ number of electrons subjected to external potential $V_{\text {ext }}(\vec{r})$ by SH equation as [5],

$$
H=\sum_{i=1}^{N}\left[-\frac{\hbar^{2} \nabla_{i}^{2}}{2 m}+V_{e x t}\left(\vec{r}_{i}\right)\right]+\sum_{i>j} \frac{e^{2}}{\left|\vec{r}_{i}-\bar{r}_{j}\right|}
$$

By knowing the solution to $\mathrm{SH}$ equation, electron density is the probability distribution of wave functions. Therefore, for inhomogeneous free electron gas, electron density $n(\vec{r})$ can be simply given based on electron wave function $(\Psi)$ as [7],

$$
n(\vec{r})=\sum_{i}\left|\Psi_{i}(\vec{r})\right|^{2}
$$

\section{Obtaining Ground State Energy by Kohn Sham Equations} [15],

According Hohenburg and Kohn, total energy $E[n]$ of any quantum mechanical system can be given by

$$
E[n]=T_{s}[n]+E_{\text {ext }}[n]+E_{H}[n]+E_{x c}[n]
$$

where, $T_{s}[n]$ is the quantum mechanical kinetic energy of electrons, $E_{\text {ext }}[n]=\int d^{3} r V_{\text {ext }}(\vec{r})$ is the energy due to external potential and mainly due to ionic cores, $E_{H}[n]$ is the classical Hartree repulsion between electrons and $E_{x c}[n]$ is the exchange correlation energy.

According to the Kohn-Sham scheme, the SH equation will be solved for single electron wave functions that only depend on three spatial variables,

$$
\left[-\frac{\hbar^{2}}{2 m} \nabla^{2}+V_{e x t}(r)+V_{H}(r)+V_{x c}(r)\right] \Psi_{i}(r)=\epsilon_{i}(r) \Psi_{i}
$$

where, $V_{\text {ext }}(\vec{r}), V_{H}(\vec{r}), V_{x c}(\vec{r})$ are the external potential, Hartree potential and exchange-correlation potential respectively. Also, $\in_{i}$ is the energy eigen value. The electron density is now obtained by single electron wave functions according to equation 2. Multiple equations depicted in equation 5 is also known as Kohn Sham equations.

Variational equation which minimizes energy with respect to electron density can be given as [15],

$$
\frac{\partial E[n]}{\partial n(\vec{r})}=\frac{\partial T_{S}[n]}{\partial n(\vec{r})}+V_{\text {ext }}(\vec{r})+e^{2} \int d^{3} r^{\prime} \frac{n(\vec{r})}{|\vec{r}-\vec{r} /|}+\frac{\partial E_{x c}[n]}{\partial n(\vec{r})}=\mu
$$

where, $\mu$ is the Lagrange multiplier associated due to conservation of total number of electrons.

Kinetic energy for virtual non-interacting system can be given as,

$$
T_{s}[n]=-\frac{\hbar^{2}}{2 m} \sum_{j} f_{j} \Psi_{j}^{*}(\vec{r}) \nabla^{2} \Psi_{j}(\vec{r})
$$

where, $f_{i}$ is the occupation orbital i with included spin degeneracy. Secondly energy due to an external potential can be obtained using $n(\vec{r})=\sum_{i} f_{i}\left|\Psi_{i}(\vec{r})\right|^{2}$, according to,

$$
E_{\text {ext }}[n]=\int n(\vec{r}) V_{\text {ext }}(\vec{r}) d r
$$

Thirdly, classical Hartree repulsion can be given taking $V_{H}(\vec{r})=\int_{\vec{r} /} \frac{n(\vec{r} /)}{|\vec{r}-\vec{r} /|} d \vec{r} /$ as,

$$
E_{H}(n)=\frac{1}{2} \int_{\vec{r}} \int_{\vec{r} /} \frac{n(\vec{r}) n(\vec{r} /)}{|\vec{r}-\vec{r} /|} d r / d r=\frac{1}{2} \int_{\vec{r}} n(\vec{r}) V_{H}(\vec{r}) d r
$$

By considering all terms, final form of Kohn-Sham total energy equation can be given as [15],

$$
E[n]=-\frac{\hbar^{2}}{2 m} \sum_{j} f_{j} \Psi_{j}^{*}(\vec{r}) \nabla^{2} \Psi_{j}(\vec{r})+\int n(\vec{r}) V_{e x t}(\vec{r}) d r+\frac{1}{2} \int_{\vec{r}} \int_{\overrightarrow{\vec{r}} /} \frac{n(\vec{r}) n(\vec{r} /)}{|\vec{r}-\vec{r} /|} d r / d r+E_{x c}
$$




\section{Exchange Correlation Energy Functional and Its Approximations}

The exchange correlation energy is decomposed to individual exchange energy $\left(E_{x}[n]\right)$ and correlation energy $\left(E_{c}[n]\right)$ as below,

$$
E_{x c}[n]=E_{x}[n]+E_{c}[n]
$$

\section{E. Local Density Approximation (LDA)}

For a uniform electron density in space, assuming $\boldsymbol{E}_{\boldsymbol{x}}$ dependent on $n(\vec{r})$ is similar as free electron gas, therefore, LDA approximation gives [15],

$$
E_{x c}^{L D A}[n]=\int n(\vec{r}) e_{x c}^{F E G}(n(\vec{r})) \cdot d^{3} r
$$

where, $e_{x c}^{F E G}(\mathrm{n}(\vec{r}))$ is the exchange correlation energy per electron in FEG, which is varying spatially.

\section{F. Generalized Gradient Approximation (GGA)}

The shortcoming of LDA is that it assumes electron density as same in based on location where, gradients of electron density is not accounted. This simplification results in in under estimation of exchange energy and over estimation of correlation energy. Spatial regions with high variation of electron density occur can be inaccurately captured by LDA approach. Therefore, to correct such tendency, the gradient of the electron density is embedded [15] as,

$$
E_{x c}^{G G A}[n, \sigma]=E_{x c}^{L D A}[n, \sigma]+\int d^{3} r e_{x c}^{G G A}(n(\vec{r}), \nabla n(\vec{r}))
$$

where, $e_{x c}^{G G A}(n(\vec{r}), \nabla n(\vec{r}))$ is the exchange correlation energy per electron in FEG, which is varying spatially as well as with respect to spatial gradient.

The main advantage of GGA approach is it considers exchange and correlation effects in small density regions more accurately compared to LDA approach.

\section{G. Application of DFT for Crystalline Solids}

Crystalline solids consist of periodic arrangement of atoms which results in periodic potentials. For free electrons in a crystalline solid, the wave function takes the from of a plane wave. Such plane wave functions can be obtained using Bloch theorem as [5],

$$
\Psi_{n k}(\vec{r})=e^{i . k \cdot \vec{r}} u_{n k}(\vec{r})
$$

where, $e^{i . k \cdot \vec{r}}$ provides the general plane wave solution which is modulated by periodic potential term $u_{n k}(\vec{r})$.

\section{H. Introduction to Pseudopotentials, Super Cells, and Periodic Boundary Conditions}

Chemical bonding and other characteristics of materials are mainly characterized by valance electrons. Pseudopotentials replace electron density from a chosen set of core electrons with a smoothed density. In pseudopotential approximation, inner electrons are considered as frozen where uncaptured and inactive electrons are assumed to be with unchanged states.

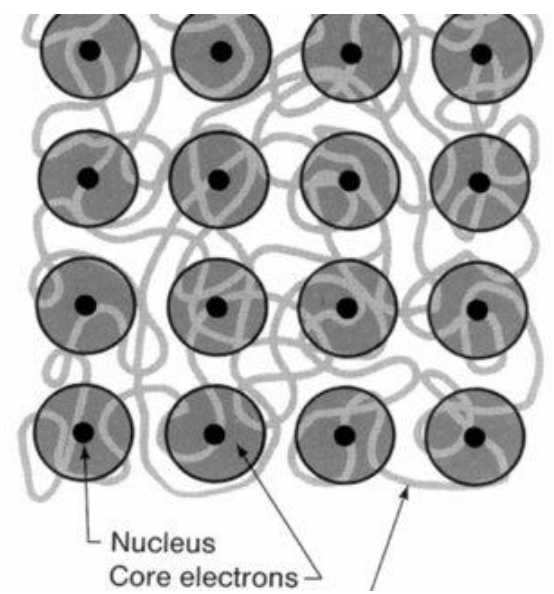

Fig. 3. Pseudopotential model for a solid with frozen core approximation. 
The unit cell applied in DFT simulations are referred to as supercells with a unique pseudopotentials. Supercell will be repeated through the entire simulation domain which results in periodic boundary conditions [16].

\section{Mehanical Property PREdiction Using DFT}

Kiely et al. [8] studied the utilization of DFT for mechanical property prediction of crystalline materials. They have demonstrated obtaining elastic properties of electroactive materials using the VASP (Vienna ab initio Simulation Package) software with using the plane wave basis set where, solution to many body SH equation is approximated in a form of a plane wave [17]. The results were experimentally validated using amino acids, peptide crystals, co-crystals and bio minerals. The calculation of elastic constant follows applying finite difference methods to obtain elastic stiffness tensor with every atom in the unit cell while displaced by a specific value $( \pm 0.01 \dot{A}$ in current case). The relative change of $\mathrm{r}$-centered $\mathrm{k}$ points with respect to atomic displacements are used for calculation of elastic constants using ELATE software. Feridoon et al. [9] utilized density functional theory to predict the mechanical properties of single wall carbon-nanotubes (SW-CNT). Their approach was to obtain elastic modulus and shear modulus of nanotubes by applying axial and torsional strains on a periodic $\mathrm{H}$ - and $\mathrm{C}$ - capped nanotube. They have utilized self-consistent charge density functional tight binding (SCC-DFTB) model to calculate total energies that results in most stable nanotube geometries. The calculation is performed for SW-CNTs of different lengths with periodic conditions along the z-axis using SCC-DFTB method. The energy of deformation as a function of carbon-carbon (C-C) bond lengths are obtained to calculate the elastic modulus.

The elastic modulus $(E M)$ was calculated using the relation [9],

$$
E M=\frac{1}{V_{0}} \frac{\partial^{2} E}{\partial^{2} \varepsilon}
$$

where, $V_{0}$ is the volume of hollow SW-CNT tube, $E$ is the deformation energy of the C-C bond length and $\varepsilon$ is the axial strain of the carbon nanotube. Similarly shear modulus $(\tau)$ can be calculated from the relation [9],

$$
\tau=\frac{1}{V_{0}} \frac{\partial E_{S}}{\partial \gamma}
$$

where, $E_{s}$ is the shear energy, and $\gamma$ is the shear strain for the entire tube.

Ajori et al. [18] also utilized DFT for mechanical property calculation of metal-free two dimensional polymer graphitic carbon-nitride $(\mathrm{g}-\mathrm{CN})$. They have utilized GGA approach to predict the exchange correlation functional. The simulation was performed with a k-point mesh with $12 \times 12$ grid points at the first Brillion zone. The elastic modulus, shear modulus and bulk modulus are calculated using similar approaches as presented by Feridoon et al. before [9].

\section{ElECTRICAL PROPERTY PREDICTION USING DFT}

Burke et al. [19] utilized DFT for obtaining electrical conductivity of molecular devices. Thy have associated Landau formalism with DFT where the model was created by placing the molecule and contacts between two infinite reservoirs at chemical potential that produces the voltage drop across the molecule. Secondly a standard integrals of Green function which is coupled to the reservoirs are utilized to simulate the current and thus predicting the conductance of the molecule. Since such simulations has to be performed in transient conditions, they have applied time dependent density functional theory (TDDFT) where, transport at finite electric fields by including dissipation to phonons. Therefore, new Kohn-Sham energy equation was derived using a new exchange correlation function [19]. Silvestrelli [12] demonstrated the use of DFT for electrical conductivity calculation using DFT for liquid sodium. They have performed the simulations using simple cubic box with 90 sodium atoms to sample the Brillion zone at five different temperatures[12]. The calculation of electrical conductivity $\left(\sigma\left(\omega, R_{I}\right)\right)$ is carried out using the major equation,

$$
\sigma\left(\omega, R_{I}\right)=\frac{2 \pi e^{2}}{3 m^{2} \omega} \frac{1}{V_{b}} \sum_{i, j}\left(f_{i}-f_{j}\right)\left|<\Psi_{i}\right| \hat{p}\left|\Psi_{j}>\right|^{\wedge} 2 \times \delta\left(E_{j}-E_{i}-\hbar \omega\right)
$$


where, $e$ and $m$ is the electronic mass and charge respectively, $V_{b}$ is the simulation box volume, $\hat{p}$ is the momentum operator and $\Psi_{i}$ and $E_{i}$ are eigenstates and energy eigenvalues obtained by DFT which is calculated for an ionic configuration $R_{I}$ at a single k-point in the first Brillion zone [12].

\section{THERMAL PROPERTY PREDICTION USING DFT}

Chena and Podloucky [11] utilized DFT for calculate thermal conductivity of thermoelectric materials $\left(\mathrm{Ba}_{8} \mathrm{Au}_{6-\mathrm{x}} \mathrm{Ge}_{40+\mathrm{x}}\right)$ with association of Lorentz number $\left(L_{0}\right)$ and Wiedemann-Franz law (WF). Secondly combination of DFT and Boltzmann transport theory has been made to obtain the thermal conductive properties. The calculations are performed assuming electron dominance of the materials. The electronic thermal conductivity $\left(k_{e l}\right)$ can be obtained using the WF law according to [11],

$$
k_{e l}=T L \sigma
$$

where, $T$ is the absolute temperature, $L$ is the Lorentz tensor and $\sigma$ is the electrical conductivity tensor. By combining with Boltzmann transport theory, for a pure metal behavior ignoring Seebeck effects, $\mathrm{L}$ can be expressed directly as [11],

$$
L=\frac{1}{e^{2} T^{2}} K_{2} K_{0}^{2}
$$

where, Lorentz tensors $\left(K_{n}\right)$ are obtained using [11],

$$
K_{n}=\frac{1}{4 \pi^{3}} \sum_{i, K} \tau_{i}(k) v_{i}(k) \otimes v_{i}(k)\left[\varepsilon_{i}(k)-\mu\right]^{\wedge} n \times\left(-\frac{\partial f\left(\mu, T, \varepsilon_{i}\right)}{\partial \varepsilon_{i}}\right)
$$

where, $\tau_{i}(k)$ is the relaxation time of electronic states with band index of $i, \varepsilon_{i}$ is the energy eigen value and $v_{i}$ is the band velocity for a wave vector k. $f(\mu, T)$ is the Fermi-Dirac distribution function with a chemical potential $\mu$ and temperature $T$. Finally $\sigma$ can also be obtained using Lorentz tensors by [11],

$$
\sigma=e^{2} K_{0}
$$

Ranasinghe et al. [14] also performed DFT to obtain thermal properties of materials for uranium dialuminide $\left(\mathrm{UAl}_{2}\right)$. Similar with the previous study [11], they have combined Boltzmann transport theory, however, now considering the phononic contributions. Phonic thermal conductivity $\left(k_{p h}\right)$ was evaluated using Boltzmann transport equation and electronic thermal conductivity $\left(k_{e l}\right)$ was obtained using the WF law as before.

For phonon dominant thermal conductivity calculations, by utilizing DFT, after obtaining phonon dispersion spectrum for the first Brillion zone, the energy of the phonons $\left(E_{p h}\right)$ can be obtained by [14],

$$
E_{p h}=\sum_{q, \vartheta} \hbar \omega_{q, \vartheta}\left[\frac{1}{2}+\frac{\exp \left(\frac{\hbar \omega_{q, \vartheta}}{k_{B} T}-1\right)}{\exp \left(\frac{\hbar \omega_{q, \vartheta}}{k_{B} T}-1\right)^{2}}\right]
$$

where, $k_{B}$ is the Boltzmann constant and $\omega_{q, \vartheta}$ is the phonon vibration frequency with a wave vector $q$ and mode $v$. The phononic energy now can be utilized to calculate the phonon specific heat of the solid as [14],

$$
C_{V}=k_{B} \sum_{q, \vartheta}\left\{\left[\frac{\hbar \omega_{q, \vartheta}}{k_{B} T}\right]^{2} \frac{\exp \left(\frac{\hbar \omega_{q, \vartheta}}{k_{B} T}-1\right)}{\exp \left(\frac{\hbar \omega_{q, \vartheta}}{k_{B} T}-1\right)^{2}}\right\}
$$

Finally, phonon thermal conductivity can be obtained using the simple relation [14],

$$
k_{p h}=\frac{1}{3} C_{V}<c>l
$$

where, $c$ is the average speed and $l$ is the mean free path of phonons.

\section{DISCUSSION AND CONCLUSION}

In summary, DFT is a well utilized molecular scale simulation tool to predict electronic as well as phononic properties of crystalline solids. DFT approaches to find a simplified solution to many body SH 
equation by utilizing electronic density as a functional. HK theory provides the main foundation to develop total energy of the quantum mechanical system as a functional of electron density. The minimization of total energy functional with respect to electron density thus provides the ground state of the system that aids in predicting energy dispersion spectra. As the most common simulation approach, pseudopotentials with plane wave approximation were utilized to obtain solutions to many body $\mathrm{SH}$ equation. Where, pseudopotentials consider core electrons are not participating in the transport process and outermost electrons as the only contribution and plane wave approximation adopted by Bloch theorem for periodic atomic arrangements.

Almost all researchers utilized DFT to obtain the electronic and phononic dispersion spectra of complex lattice arrangement where, solution from many body $\mathrm{SH}$ equation is not feasible. Obtained dispersion spectra can be further analyzed to obtain electronic and phonon density of states to calculate electronic and thermal properties (electrical conductivity, specific heat capacity, thermal conductivity). More importantly, knowing the ground state energy of the quantum mechanical system, it can be further utilized to obtain mechanical properties of the crystalline material based on interatomic displacements.

\section{ACKNOWLEDGMENT}

Authors pay their gratitude for Dr. Laksman Perera (Emeritus) of Department of Physics and Astronomy of University of Colombo, Sri Lanka for constatntly supporting this work.

\section{CONFLICT OF INTEREST}

Authors declare that they do not have any conflict of interest.

\section{REFERENCES}

[1] H. Ma, Y. Ma, and Z. Tian. Simple Theoretical Model for Thermal Conductivity of Crystalline Polymers. ACS Appl. Polym. Mater., Oct. 2019;1(10):2566-2570. doi: 10.1021/acsapm.9b00605.

[2] N. A. Mohd Radzuan, A. B. Sulong, and J. Sahari. A review of electrical conductivity models for conductive polymer composite. Int. J. Hydrog. Energy, Apr. 2017;42(14):9262-9273. doi: 10.1016/j.ijhydene.2016.03.045.

[3] F. Willems and C. Bonten. Prediction of the mechanical properties of long fiber reinforced thermoplastics. Mallorca, Spain, 2020, p. 020066. doi: 10.1063/5.0028790.

[4] P. E. Hopkins, M. Ding, and J. Poon. Contributions of electron and phonon transport to the thermal conductivity of GdFeCo and TbFeCo amorphous rare-earth transition-metal alloys. J. Appl. Phys., May 2012;111(10):103533, doi: 10.1063/1.4722231.

[5] Eisberg, Robert Martin. Quantum physics of atoms, molecules, solids, nuclei, and particles. Wiley, 1985.

[6] K. Pal, S. Anand, and U. V. Waghmare. Thermoelectric properties of materials with nontrivial electronic topology. J. Mater. Chem. C, 2015;3(46):12130-12139. doi: 10.1039/C5TC02344K.

[7] N. M. Harrison. An Introduction to Density Functional Theory, p. 26.

[8] E. Kiely, R. Zwane, R. Fox, A. M. Reilly, and S. Guerin. Density functional theory predictions of the mechanical properties of crystalline materials. CrystEngComm, 2021;23(34):5697-5710. doi: 10.1039/D1CE00453K.

[9] A. Fereidoon, M. Ghorbanzadeh Ahangari, M. D. Ganji, and M. Jahanshahi. Density functional theory investigation of the mechanical properties of single-walled carbon nanotubes. Comput. Mater. Sci., Feb. 2012;53(1):377-381. doi: 10.1016/j.commatsci.2011.08.007.

[10] S. Ajori. A density functional study on the mechanical properties of metal-free two dimensional polymer graphitic Carbon Nitride, Int.L. of Nano Dimensions, 2017;8(3), 234-240.

[11] M. X. Chen and R. Podloucky. Electronic thermal conductivity as derived by density functional theory. Phys. Rev. B, Jul. 2013;88(4):045134. doi: 10.1103/PhysRevB.88.045134.

[12] P. L. Silvestrelli, A. Alavi, and M. Parrinello. Electrical-conductivity calculation in ab initio simulations of metals:Application to liquid sodium. Phys. Rev. B, Jun. 1997;55(23):15515-15522. doi: 10.1103/PhysRevB.55.15515.

[13] J. Bardeen. Electrical Conductivity of Metals. J. Appl. Phys., 1940;11:25.

[14] J. I. Ranasinghe, L. Malakkal, E. Jossou, B. Szpunar, and J. A. Szpunar. Density functional theory study of the structural, mechanical and thermal conductivity of uranium dialuminide (UA12). J. Nucl. Mater., Nov. 2020;540:152359. doi: 10.1016/j.jnucmat.2020.152359.

[15] K. A. Baseden and J. W. Tye. Introduction to Density Functional Theory: Calculations by Hand on the Helium Atom. J. Chem. Educ., Dec. 2014;91(12):2116-2123. doi: 10.1021/ed5004788.

[16] P. Borlido, J. Doumont, F. Tran, M. A. L. Marques, and S. Botti. Validation of Pseudopotential Calculations for the Electronic Band Gap of Solids. J. Chem. Theory Comput., Jun. 2020;16(6):3620-3627. doi: 10.1021/acs.jctc.0c00214.

[17] G. Aulakh. An overview of density functional theory based codes used for ab initio calculations, p. 10.

[18] K. Burke, R. Car, and R. Gebauer. Density Functional Theory of the Electrical Conductivity of Molecular Devices. Phys. Rev. Lett., Apr. 2005;94(14):146803. doi: 10.1103/PhysRevLett.94.146803.

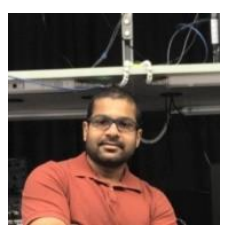

Naveen Weerasekera is a doctoral candidate at Department of Mechanical Engineering of University of Louisville, Louisville, KY, USA. He has completed is Master's degree in mechanical engineering from Portland State Univeristy, Portland, OR. He is currently working as a departmental research assistant at University of Louisville under multiple fellowships. His research interests falls to solid state refrigeration devices and evaluating material properties of functional materials for efficient caloric cooling. He utilizes experimental, analytical as well as numerical approachs to asses functional properties of materials. 
Siyua Cao is a doctoral candidate at Department of Mathematics and Statistics of Portland State University, Portland, OR. He has completed is master's degree in mathematics from Portland State University. He currently works as a research and teaching assistant at Portland State University. His research interests fall on advanced approaches to obtain solutions to many-body Schrodinger equation by numerical approaches. He utilizes statistical as well as numerical approaches to conduct his research.

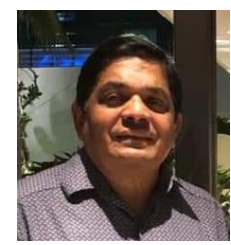

Laksman Perera is an emeritus adjunct faculty of Department of Physics and Astronomy of University of Colombo, Sri Lanka. His research interests include advanced electronics in quantum well infrared photo detectors. He worked as a former director in Upali electronics radio frequency appliances division. 